\title{
X-RAY PROPERTIES OF EARLY-TYPE STARS
}

\author{
THOMAS W. BERGHÖFER and JÜRGEN H. M. M. SCHMITT \\ Max-Planck-Institut für extraterrestrische Physik \\ Postfach 1603 D-85740 Garching
}

\section{Introduction}

Extensive stellar surveys with the Einstein Observatory (Chlebowski et al., 1989 ) and with ROSAT have clearly confirmed the presence of stellar X-ray emission over nearly the whole range of the HR diagram. In the ROSAT all-sky survey data approximately 20000 stellar X-ray sources were detected (Schmitt et al., 1992). Most of these stellar X-ray emitters are low mass late-type stars, the origin of their X-ray emission is thought to be coronal.

In the case of the early-type stars, the X-ray emission is thought to be generated by shock-heated gas (Lucy and White, 1980), present in the strong stellar winds of these stars. Model calculations for radiatively driven winds show that instabilities lead to the production of strong shocks and hence to the X-ray emission in the stellar wind (e.g. Owocki et al., 1988).

All the models for X-ray production in early-type star are based on assumptions for the underlying shock structures to heat the $\mathrm{X}$-ray emitting gas. The search for variability in X-ray emission of early-type stars is a check for the so far assumed shock structures and is the only way to get access to parameters like the cooling time and the occurrence rate of shocks.

\section{ROSAT observations and results}

During the ROSAT all-sky survey (RASS) each star was observed for at least 2 days. We selected all detected early-type OB stars (single stars, not X-ray binaries!) with an X-ray count rate greater than 0.1 counts/s and listed in the Bright Star Catalog. For this magnitude and flux limited sample of early-type stars, (57 OB-type stars (04-B3)), we generated the RASS X-ray light curves and tested them for variability. As an example we present in Fig. 1 the RASS light curves of the two $O$ stars $\zeta$ Ori and $\iota$ Ori. The RASS X-ray light curves of our 57 OB stars show no significant variations above the $3 \sigma$ level, and thus for 2 days these stars must have had an intrinsic memory of how much X-ray output to produce.

For a consistency check of the Einstein (Chlebowski et al., 1989, Grillo et al., 1992) and RASS data as well as a search for variability on a time scale of approximately 10 years we compared the Einstein and RASS X-ray count rates. For most of our OB stars the relation of the Einstein and RASS $\mathrm{X}$-ray count rates is compatible with the relation of the effective area of the PSPC (ROSAT) and the IPC (Einstein). However, in the case of $4 \mathrm{~B}$ stars we observe, compared to the respective Einstein values, an overluminous RASS X-ray count rate. 


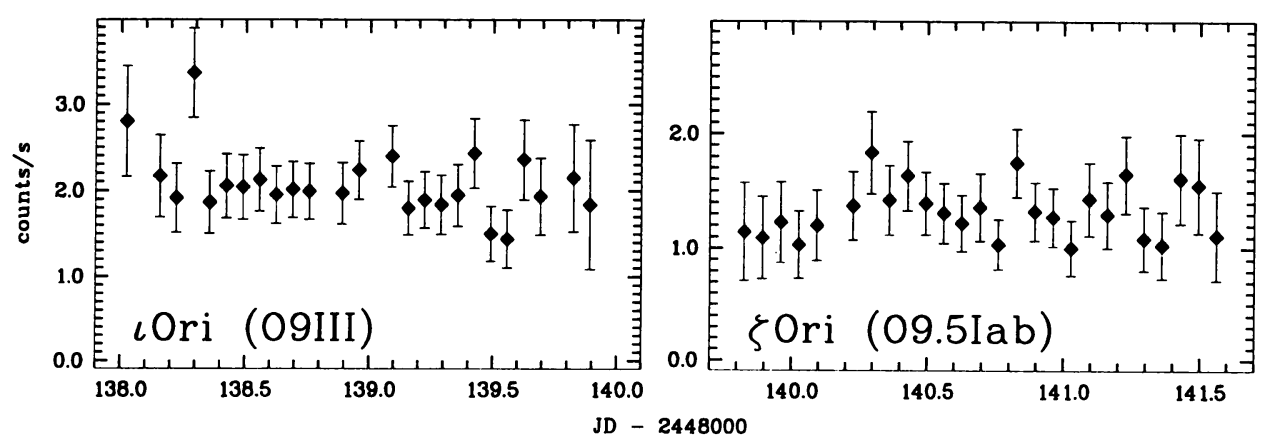

Fig. 1. ROSAT all-sky survey light curves of the two $O$ stars $\iota$ Ori and $\zeta$ Ori.
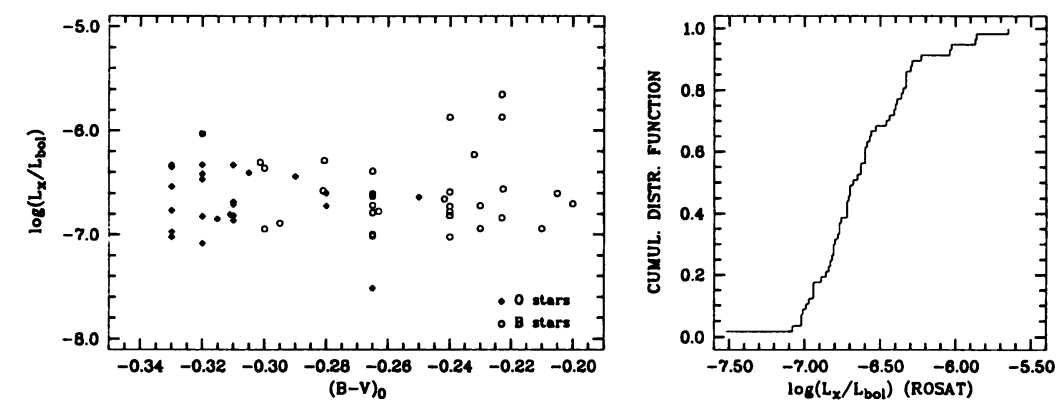

Fig. 2. Plot of the computed $L_{x} / L_{b o l}$ values as a function of $(B-V)_{0}$ and the respective cumulative distribution function

As a result of the Einstein observations (Long and White, 1980), it is established that X-ray emission of OB stars obeys the relation $\mathrm{L}_{\mathbf{x}} / \mathrm{L}_{\mathrm{bol}} \approx$ $10^{-7}$. For stars later than B5 this relation breaks down and is definitely not valid for A-type stars (Schmitt et al., 1985). We have started to compute the relation $\mathrm{L}_{\mathbf{x}} / \mathrm{L}_{\text {bol }}$ for all detected $\mathrm{OB}$ stars. As a preliminary result we present for our sample of $O B$ stars the $L_{x} / L_{b o l}$ values. For the bulk portion of our program stars $(90 \%)$ we compute a $\log \left(\mathrm{L}_{\mathbf{x}} / \mathrm{L}_{\mathrm{bol}}\right)$ value of $-6.7 \pm 0.4 \mathrm{dex}$. In the case of the 0 stars of our sample, the $\log \left(\mathrm{L}_{\mathbf{x}} / \mathrm{L}_{\mathrm{bol}}\right)$ values agree with the respective Einstein values. However, some of the B stars appear to be overluminous compared to the observation with the Einstein Observatory.

\section{References}

Chlebowski, T., Harnden, F.R., Jr. and Sciortino, S.: 1989, Astrophys. J.341, 427

Grillo, F., et al.: 1992, Astrophys. J. Suppl. Ser.81, 795

Long, K.S., White, R.L.: 1980, Astrophys. J.239, L65

Lucy, L.B., White, R.L.: 1980, Astrophys. J.241, 300

Owocki, S.P., Castor, J.L., Rybicki, G.B.: 1988, Astrophys. J.335, 915

Schmitt J.H.M.M., et al.: 1985, Astrophys. J.290, 307

Schmitt, J.H.M.M., Kahabka, P., Stauffer, J., Piters, A.: 1993, Astron. Astrophys.277, 114 